\title{
Outpatient Treatment of Lower Respiratory Tract Infections in Children with Tracheostomy
}

\author{
Parevi Majmudar ${ }^{1}$, Don Sanders ${ }^{1}$, Andrew Beardsley ${ }^{1}$, James Slaven ${ }^{2}$, and A. Ioana \\ Cristea $^{1}$ \\ ${ }^{1}$ Riley Hospital for Children at Indiana University Health \\ ${ }^{2}$ Indiana University School of Medicine
}

June 26, 2020

\begin{abstract}
RATIONALE: Outpatient treatment of lower respiratory tract infection (LRTI) in tracheostomy dependent children varies institutionally. The objective of this study was to identify whether only increasing airway clearance (AWC) increased the odds of hospitalization within 28 days of treatment. Our hypothesis was that those treated with antibiotics were less likely to be hospitalized. METHODS: We retrospectively reviewed medical charts of children who were tracheostomy dependent between 2012-2019 and followed at our institution. We recorded recommendations with each sick call, i.e. prescription of antibiotics and/or increase in frequency of airway clearance. Generalized estimating equation models were used to determine whether the recommendation to increase AWC frequency was associated with an increased risk of hospitalization within 4 weeks, as compared to the prescription of oral and/or inhaled antibiotics. RESULTS: Of the eighty -two patients reviewed, there were 283 unique episodes of LRTI. 160 (45\%) episodes involved increasing AWC alone and 195 (55\%) were given an antibiotic in addition to increasing AWC. Of those who received AWC only, 21.7\% were hospitalized within 28 days of treatment, and $13.8 \%$ were hospitalized after treatment with increased AWC and oral/inhaled antibiotics, $\mathrm{p}=0.08$. Those who received only AWC did not have significantly higher odds of hospitalization within 28 days of treatment, compared to those who received an antibiotic: adjusted OR 1.47 (95\% CI: 0.75, 2.86); p=.26. CONCLUSIONS: In this retrospective cohort study of pediatric patients with tracheostomy, a recommendation to increase airway clearance only versus initiating an antibiotic was not associated with increased odds of hospitalization.
\end{abstract}

Title: Outpatient Treatment of Lower Respiratory Tract Infections in Children with Tracheostomy

Authors: Parevi Majmudar DO ${ }^{1}$, D.B. Sanders $\mathrm{MD}^{1}$, Andrew Beardsley MD ${ }^{2}$, James Slaven $\mathrm{MS}^{3}$, A. Ioana Cristea $\mathrm{MD}^{1}$

${ }^{1}$ Division of Pulmonary, Allergy and Sleep Medicine, Riley Hospital for Children at Indiana University Health, Indianapolis, IN

${ }^{2}$ Division of Pediatric Critical Care Medicine, Riley Hospital for Children at Indiana University Health, Indianapolis, IN

${ }^{3}$ Department of Biostatistics, Indiana University School of Medicine, Indianapolis, IN

Funding: None.

Meetings: This work will be presented at the virtual American Thoracic Society meeting in 2020.

Correspondence: Parevi Majmudar, 705 Riley Hospital Dr, ROC 4270, Indianapolis, IN 46202

Telephone: 317-948-0949, Fax: 317-944-5791 


\section{Email: Parevipm@gmail.com}

Keywords: tracheitis, inhaled tobramycin, hospitalization, complex medical conditions

Abstract

RATIONALE: Outpatient treatment of lower respiratory tract infection (LRTI) in tracheostomy dependent children varies. The objective of this study was to identify whether increasing frequency of airway clearance (AWC) alone increased the odds of hospitalization within 28 days of treatment. Our hypothesis was that those treated with antibiotics were less likely to be hospitalized.

METHODS: We reviewed medical records of children who were tracheostomy dependent between 2012-2019 and followed at our institution. We recorded recommendations provided at each sick call or clinic visit, i.e. prescription of antibiotics and/or increase in frequency of AWC, for physician defined LRTI. Generalized estimating equation models were used to determine whether the recommendation to increase AWC frequency was associated with an increased risk of hospitalization within 4 weeks, as compared to the prescription of oral and/or inhaled antibiotics.

RESULTS: Among the 82 patients reviewed, there were 283 unique episodes of LRTI. Increasing AWC alone was recommended for $160(45 \%)$ episodes and antibiotics were prescribed for $195(55 \%)$ in addition to increasing AWC. Of those who received AWC only, 21.7\% were hospitalized within 28 days of treatment, compared to $13.8 \%$ prescribed oral/inhaled antibiotics, $\mathrm{p}=0.08$. Those who received AWC alone did not have significantly higher odds of hospitalization within 28 days of treatment: adjusted OR 1.47 (95\% CI: $0.75,2.86) ; \mathrm{p}=.26$.

CONCLUSIONS: In this retrospective cohort study of pediatric patients with tracheostomy, a recommendation to increase AWC alone was not associated with increased odds of hospitalization compared to antibiotic prescriptions.

\section{Introduction}

An increasing number of critically ill children are surviving to hospital discharge and tracheostomy placement has become more widespread, often serving as a bridge from hospital to home. In addition to tracheostomy, these children often have other comorbidities requiring technology assistance including ventilators, gastrostomy tubes, vagal nerve stimulators and cerebrospinal fluid shunts ${ }^{1}$.

Despite the benefits of tracheostomy, rehospitalization is common, often due to respiratory illness, major surgery, technology malfunction, seizure or feeding difficulty ${ }^{1}$. Hospitalizations for children with tracheostomy account for over $\$ 2.6$ billion each year ${ }^{2}$. In 2019 , the readmission rate for children on home mechanical ventilation was 2.16 hospitalizations per patient in the first year following discharge and average readmission cost was $\$ 73,144$ per patient ${ }^{3}$. The length of stay was increased in those with complex comorbidities and in those who were younger at time of admission ${ }^{2,4}$. Respiratory diagnoses are still the most frequent indications for admission ${ }^{2}$, accounting for more than 2000 admissions and $\$ 100$ million in hospital costs in $2009^{4}$.

Considering this high healthcare burden on families and resource utilization, every effort is made to treat respiratory infections safely at home. Even though there is a growing number of children with tracheostomy, there is little published evidence regarding their chronic care including identification and management of respiratory tract infections ${ }^{5}$. Since there are no guidelines for home treatment of respiratory tract infections, choice of therapy varies by institution and is practitioner driven.

The most common outpatient interventions include antibiotics (oral or inhaled) and increasing frequency of airway clearance (AWC). In the outpatient setting, it is often unclear if a patient would benefit from antibiotics, and benefits must be weighed against risk associated with antibiotics such as cost, drug-related side effects, and antibiotic resistance. Due to the lack of natural upper airway humidification and thicker secretions of patients with tracheostomy, providers may recommend an increase in AWC therapies in an attempt to improve mucus clearance and prevent or remedy infection. 
Currently, there are no studies that evaluate the incidence of hospitalization after outpatient treatment for lower respiratory tract infections (LRTI) in children with tracheostomy. The objective of this study was to identify whether there was a difference in risk of hospitalization for LRTI within 28 days of treatment comparing antibiotic prescription to increasing AWC alone. Our hypothesis was that those treated with antibiotics were less likely to be hospitalized following treatment.

\section{Methods}

This was a single center retrospective study of patients with tracheostomy who were followed at Riley Hospital for Children at Indiana University Health from January 2012- December 2019. Eighty-two patients were identified through review of internal tracheostomy and chronic ventilator patient lists. Patients were included in the study if they were tracheostomy dependent anytime between 2012-2019 and received pulmonary outpatient care at Riley Hospital for Children. Patients were excluded if they had a diagnosis of cystic fibrosis, primary ciliary dyskinesia or malignancy, lived primarily at a long term care facility or if they did not have any sick calls for respiratory illness.

For each patient, we reviewed all outpatient clinic visits, phone messages recorded in the electronic medical record, emergency room (ER) visits, and respiratory hospitalizations at Riley Hospital for Children or other hospitals within the Indiana University Health system. We gathered demographic data including sex, age and race. Tracheostomy information including date of placement, date of initial hospital discharge, date of ventilator liberation, and date of decannulation. Respiratory cultures were collected via tracheal aspirate, blind bronchoalveolar lavage (BAL) or bronchoscopic BAL were recorded. Blind BAL samples are obtained by introducing a lavage catheter into the artificial airway. The catheter is advanced into the distal airway until gentle resistance is met. $0.5 \mathrm{~mL} / \mathrm{kg}$ of $0.9 \% \mathrm{NaCl}$ is then instilled through the catheter and aspirated into the same syringe. Most of the cultures were collected while the patient was admitted for illness. Routine surveillance cultures are not part of the standard of care at our institution.

Outpatient treatment of respiratory tract infections involved increasing frequency and/or intensity of routine AWC measures. Such measures included chest physiotherapy via manual percussion or high-frequency chest wall compression vests, cough assist and nebulized inhalation of albuterol and $3 \%$ sodium chloride. Treatment may also include enteral or inhaled antibiotics in addition to increasing AWC, which are usually selected based on patient symptoms and clinician judgement. We defined a treatment episode as a recommendation to increase AWC and/or begin an antibiotic in the outpatient setting (i.e. via phone or during a clinic visit) for treatment of an LRTI. LRTI was physician defined and included diagnoses of tracheitis, tracheobronchitis or pneumonia and/or symptoms of cough, increased work of breathing, fever, or change in tracheostomy secretions. Details of each treatment episode were recorded including date of treatment, patient symptoms and whether increased AWC only or antibiotics and increased AWC were recommended by the practitioner. Additional treatment episodes within a 28 day period were not included if the encounter resulted in the same intervention. Additional episodes were included if the treatment method changed.

\section{Statistical analysis}

Demographic and clinical characteristics were compared between the treatment groups in a bivariate manner, using Wilcoxon rank-sum tests for continuous variables, due to data skewness, and Chi-Square tests for categorical data, using Fisher's Exact when expected cell counts were small.

The primary outcome was hospitalization. If a hospitalization occurred within 28 days of an outpatient treatment episode, it was counted as a failure of that treatment. Generalized Estimating Equations (GEE) models were used to determine the association between treatment (AWC alone versus any antibiotics) and hospitalization. Unadjusted and adjusted models were both performed, with the covariates in the multivariable adjusted model being chosen based on their clinical relevance: hospitalization within the past three months of a treatment episode, any prior respiratory culture positive for Pseudomonas aeruginosa , indication for tracheostomy (prematurity, neuromuscular disease and airway obstruction) and time since tracheostomy placement. 
All analytic assumptions were verified and analyses were performed using SAS v9.4 (SAS Institute, Cary, NC). This study was approved by Indiana University Institutional Review Board. All data was stored on a REDCap database.

Results

Patient and treatment characteristics

Eighty- two patient charts were reviewed. Ten patients did not have any treatment episodes and were not included in the study. Baseline demographic and clinical characteristics are outlined in Table 1. There were 355 treatment episodes amongst 72 patients over the 7 year study period. The majority of patients were white and male. The most common indications for tracheostomy were prematurity and neuromuscular disease. Sixty-four of the 72 patients were ventilator dependent at some point over the course of this study. Two patients died during the study period with median age at death of 6 years.

Of the 355 treatment episodes, 160 (45\%) episodes involved increasing AWC alone and 195 (55\%) were given an antibiotic in addition to increasing AWC. Inhaled tobramycin was the most commonly prescribed antibiotic, followed by amoxicillin-clavulanate (Table 2).Pseudomonas aeruginosa was the most common species on respiratory culture followed by Stenotrophomonas maltophilia and then methicillin resistant Staphylococcus aureus (MRSA) (Table 3). Those who cultured $P$. aeruginosa at any time were more likely to be treated with inhaled tobramycin compared to other outpatient antibiotics. Patients who never cultured $P$. aeruginosa were often treated with anti-pseudomonal therapy. Children who ever cultured S. maltophilia were often treated with antibiotics that did not provide coverage.

\section{Risk of hospitalization}

Of the 355 treatment episodes, 72 episodes represented retreatment with the same treatment method within a 28 day period so these were not included in the analysis. Of the 283 unique treatment episodes that remained, $21.7 \%$ of those who received increased frequency of AWC only were hospitalized within 28 days of treatment, compared to $13.8 \%$ that were hospitalized after treatment with increased airway clearance and oral/inhaled antibiotics, $\mathrm{p}=0.08$. The unadjusted odds ratio for hospitalization was 1.73 (95\% CI: $0.88,3.42) ; \mathrm{p}=0.11$, for treatment episodes treated with AWC alone compared to treatment episodes treated with AWC and antibiotics. In a multivariable model adjusting for important clinical covariates, the odds of hospitalization remained non-significant, adjusted OR 1.47 (95\% CI: 0.75, 2.86) (Table 4). Among the selected covariates, hospitalization within the previous three months of a sick call was associated with reduced odds of rehospitalization.

\section{Discussion}

There is substantial variability in the surveillance and management of children with tracheostomy. Clinical guidelines are lacking in several aspects of tracheostomy care, including outpatient management of respiratory infections. Hospitalizations for these patients are lengthy, costly and common. To our knowledge, this is the first study to describe the outpatient treatment of respiratory infections, as well as to examine the impact of hospitalizations on children with long term tracheostomy.

In this study, we retrospectively analyzed whether hospitalization rates due to LRTI in patients with tracheostomy were affected by outpatient treatment methods of either receiving only increased AWC or receiving an antibiotic in addition to increased AWC. We found that those who received only AWC did not have significantly higher odds of hospitalization within 28 days of treatment, compared to those who received an antibiotic. This data indicates that there may be no advantage to prescribing antibiotics. Antibiotics can be costly, can induce bacterial resistance and can have systemic side effects, even in the case of inhaled tobramycin as renal failure and ototoxicity have been reported ${ }^{6-8}$.

We identified no studies that evaluated the same interventions and outcome. However a study by Zhu ${ }^{4}$ investigated hospitalizations due to ambulatory care sensitive conditions (ACSCs) which were defined as conditions for which good outpatient care could potentially prevent the need for hospitalization or for which 
early intervention could prevent complications. They found the most frequent and costly ACSCs that lead to hospitalization were bacterial pneumonia and tracheostomy infection. This study, along with our study where we found that patients called frequently for respiratory concerns and were treated often for these illnesses, indicates a need to identify optimal treatments to effectively treat LRTIs at home.

As noted earlier in the study, inhaled tobramycin was the most commonly prescribed antibiotic. Anecdotally, many parents prefer inhaled tobramycin due to its localized activity and fewer systemic side effects such as diarrhea and abdominal upset. In the absence of evidence based guidelines for treatment of LRTI in this patient population, antibiotic choice was determined by previous respiratory culture results, patient/parent preference, accessibility to and availability of the medication, insurance coverage and affordability. The intravenous form of tobramycin for inhalation is not widely available in outpatient pharmacies, with enteral antibiotics often being prescribed in its place.

Despite minimal data, treatment of LRTI is often based on previous cultures or most common bacteria cited in the literature ( $P$. aeruginosa and MRSA $)^{5}$. In agreement with previous studies, we found $P$. aeruginosa to be the most commonly isolated pathogen on respiratory cultures ${ }^{9,10}$. This is in part due to the tenacious nature of Pseudomonas which strongly adheres to the tracheal mucosa ${ }^{9}$. Though there is conflicting evidence, several authors have shown MRSA to also be prevalent in tracheal cultures, second to P. aeruginosa ${ }^{9,10}$. Nevertheless this was not the case with our study where $S$. maltophilia was actually more common than MRSA.

The cystic fibrosis (CF) literature has demonstrated an association between $P$. aeruginosa acquisition and increased pulmonary exacerbation rates, declining lung function and greater mortality ${ }^{11-13}$, as well as shortened survival in those with MRSA ${ }^{14}$. The acquisition and outcomes of eradication of P. aeruginosa and MRSA in pediatric patients with tracheostomy remains unclear though a retrospective study by Russell ${ }^{15}$ showed that acquisition of $P$. aeruginosa was associated with significantly increased odds of readmission for respiratory tract infections within 12 months of discharge from the tracheostomy placement hospitalization.

While obtaining routine respiratory cultures is the norm in patients with cystic fibrosis ${ }^{16,17}$, there is no evidence showing the benefit of collecting surveillance respiratory cultures in healthy patients with tracheostomy. Biofilm is known to develop in tracheostomy tubes as soon as 7 days after tracheostomy tube change or initial placement ${ }^{18}$ and results in subsequent bacterial colonization of the airway. Cline et al ${ }^{19}$ showed that bacteria and antibiotic sensitivity can change between consecutive respiratory cultures and thus use of prior cultures was of limited benefit for choosing antibiotic therapy in treating exacerbations. In addition, reports have shown that colonization without infection is common and there are no good methods for distinguishing colonization (which may not require treatment) from overt infection that requires intervention ${ }^{20,21}$.

Nevertheless, many practitioners use prior respiratory cultures as a guide for treatment of LRTI ${ }^{5}$. However, despite the prevalence of $S$. maltophilia in our patients' respiratory cultures, trimethoprim-sulfamethoxazole was not prescribed as often as would be expected. This could be because S. maltophilia may not have grown on the most recent culture and so was not thought to be the cause of the current LRTI. We also think it could be due to previous findings showing that most children with tracheosomy will acquire $P$. aeruginosa ${ }^{10,15}$ and would benefit from anti-pseudomonal targeted therapy. A recent study ${ }^{22}$ evaluated treatment of $S$. maltophilia in children with tracheostomy and acute respiratory illness. They found no difference in the amount of time to return to respiratory baseline after treatment targeting S. maltophilia with trimethoprim-sulfamethoxazole versus those who were also $S$. maltophilia positive but did not receive targeted treatment. This study shows there may be no benefit of targeted treatment of S. maltophilia in this group, but more rigorous evaluation is needed.

There were several limitations to this study. This is a retrospective study, so we cannot ascribe causality to interventions for LRTI. Our sample size was small and does not preclude type II error, i.e., that antibiotics are effective in reducing LRTI hospitalizations in children with tracheostomy. Additionally, most of the patients were young and received a tracheostomy for prematurity and neuromuscular disease. Further study is required for children over 6 years of age as well as patients with other indications for tracheostomy. 
We accounted for four confounders that we thought most impacted outcomes including lower odds of hospitalization if patients were recently hospitalized; however there were other confounders that we did not evaluate such as socioeconomic status, smoke exposure at home, daycare or school attendance and home nursing since this data was missing or incomplete. Cristea et al ${ }^{23}$ showed that chronically ventilated children who lived in lower ZIP code- based annual household income areas had higher mortality.

Next, several patients also received treatment outside of Riley Hospital (such as antibiotics for LRTI from primary care provider) and we were only aware of these treatment episodes if parents called our clinic to report it. Our study was focused on chronically ventilated patients as these patients were more likely to be treated solely within our hospital system compared to patients who were not chronically ventilated, ensuring that we were less likely to miss any episodes of LRTI. It is reasonable to assume that we underestimated the number of treatment episodes given outside of Riley Hospital considering it was easier to see the local pediatrician than our outpatient clinic for a sick visit either due to distance or sick visit availability. Similarly, patients were sometimes hospitalized outside of Riley Hospital. We were only aware of the hospitalizations if discharge paperwork was sent to us and so the number of hospitalizations was also likely underestimated. Our analysis does not account for adherence to the prescribed treatments. Diagnosis of LRTI lacks consistency owing to various definitions of infection and treatment recommendations, further complicating decision to treat with antibiotics ${ }^{24,25}$.

\section{Conclusions}

Children with tracheostomy represent a vulnerable population of patients who often have multiple complex co-morbidities and have high level of resource utilization and antibiotic exposure. Our study shows that these children are frequently treated for respiratory illness. Successfully treating illnesses at home would minimize healthcare costs and avoid hospitalizations. We did not find a difference in subsequent 28 day hospitalization between starting outpatient antibiotics versus only increasing AWC, indicating that antibiotics may not help with avoiding hospitalization and should be used only after careful consideration of the patient's clinical status and medical history. Prospective studies to determine optimal outpatient treatment strategies are needed.

References

1. Russell CJ, Simon TD. Care of children with medical complexity in the hospital setting. Pediatric annals 2014;43:e157-e62.

2. Russell CJ, Mack WJ, Schrager SM, Wu S. Care variations and outcomes for children hospitalized with bacterial tracheostomy-associated respiratory infections. Hospital pediatrics 2017;7:16-23.

3. Rogerson CM, Beardsley AL, Nitu ME, Cristea AI. Health Care Resource Utilization for Children Requiring Prolonged Mechanical Ventilation via Tracheostomy. Respiratory Care 2020.

4. Zhu H, Das P, Roberson DW, et al. Hospitalizations in children with preexisting tracheostomy: a national perspective. The Laryngoscope 2015;125:462-8.

5. Rusakow LS, Guaría M, Wegner CB, Rice TB, Mischler EH. Suspected respiratory tract infection in the tracheostomized child: the pediatric pulmonologist's approach. Chest 1998;113:1549-54.

6. Edson RS, Brey RH, McDonald TJ, Terrell CL, McCarthy JT, Thibert JM. Vestibular toxicity due to inhaled tobramycin in a patient with renal insufficiency. Mayo Clinic Proceedings; 2004: Elsevier. p. 1185-91.

7. Kahler DA, Schowengerdt KO, Fricker FJ, Mansfield M, Visner GA, Faro A. Toxic serum trough concentrations after administration of nebulized tobramycin. Pharmacotherapy: The Journal of Human Pharmacology and Drug Therapy 2003;23:543-5.

8. Patatanian L. Inhaled tobramycin-associated hearing loss in an adolescent with renal failure. The Pediatric infectious disease journal 2006;25:276-8. 
9. Niederman MS, Ferranti RD, Zeigler A, Merrill WW, Reynolds HY. Respiratory infection complicating long-term tracheostomy: the implication of persistent gram-negative tracheobronchial colonization. Chest 1984;85:39-44.

10. McCaleb R, Warren RH, Willis D, Maples HD, Bai S, O'Brien CE. Description of respiratory microbiology of children with long-term tracheostomies. Respiratory care 2016;61:447-52.

11. Henry RL, Mellis CM, Petrovic L. Mucoid Pseudomonas aeruginosa is a marker of poor survival in cystic fibrosis. Pediatric pulmonology 1992;12:158-61.

12. Zemanick ET, Emerson J, Thompson V, et al. Clinical outcomes after initial pseudomonas acquisition in cystic fibrosis. Pediatric pulmonology 2015;50:42-8.

13. Kosorok MR, Zeng L, West SE, et al. Acceleration of lung disease in children with cystic fibrosis after Pseudomonas aeruginosa acquisition. Pediatric pulmonology 2001;32:277-87.

14. Dasenbrook EC, Checkley W, Merlo CA, Konstan MW, Lechtzin N, Boyle MP. Association between respiratory tract methicillin-resistant Staphylococcus aureus and survival in cystic fibrosis. Jama 2010;303:238692.

15. Russell CJ, Simon TD, Mamey MR, Newth CJ, Neely MN. Pseudomonas aeruginosa and posttracheotomy bacterial respiratory tract infection readmissions. Pediatric pulmonology 2017;52:1212-8.

16. Mogayzel Jr PJ, Naureckas ET, Robinson KA, et al. Cystic Fibrosis Foundation pulmonary guideline. Pharmacologic approaches to prevention and eradication of initial Pseudomonas aeruginosa infection. Annals of the American Thoracic Society 2014;11:1640-50.

17. Rosenfeld M, Emerson J, Accurso F, et al. Diagnostic accuracy of oropharyngeal cultures in infants and young children with cystic fibrosis. Pediatric pulmonology 1999;28:321-8.

18. Solomon DH, Wobb J, Buttaro BA, Truant A, Soliman AM. Characterization of bacterial biofilms on tracheostomy tubes. The Laryngoscope 2009;119:1633-8.

19. Cline JM, Woods CR, Ervin SE, Rubin BK, Kirse DJ. Surveillance tracheal aspirate cultures do not reliably predict bacteria cultured at the time of an acute respiratory infection in children with tracheostomy tubes. Chest 2012;141:625-31.

20. Bartlett JG, Faling LJ, Willey S. Quantitative tracheal bacteriologic and cytologic studies in patients with long-term tracheostomies. Chest 1978;74:635-9.

21. Morar P, Singh V, Jones AS, Hughes J, Van Saene R. Impact of tracheotomy on colonization and infection of lower airways in children requiring long-term ventilation: a prospective observational cohort study. Chest 1998;113:77-85.

22. Tillman EM, Firmani SE, Ackerman VL, Slaven JE, Cristea AI. Evaluation of the Treatment of Stenotrophomonas maltophilia in Tracheostomy-Dependent Pediatric Patients. The Journal of Pediatric Pharmacology and Therapeutics 2019;24:510-6.

23. Cristea AI, Ackerman VL, Davis SD, et al. Median household income: association with mortality in children on chronic ventilation at home secondary to bronchopulmonary dysplasia. Pediatric allergy, immunology, and pulmonology 2015;28:41-6.

24. Beardsley AL, Nitu ME, Cox EG, Benneyworth BD. An evaluation of various ventilator-associated infection criteria in a PICU. Pediatric Critical Care Medicine 2016;17:73-80.

25. Willson DF, Hall M, Beardsley A, et al. Pediatric Ventilator-Associated Events: Analysis of the Pediatric Ventilator-Associated Infection Data. Pediatric critical care medicine 2018;19:e631-e6.

\section{Hosted file}


Manuscript tables.docx available at https://authorea.com/users/337200/articles/462808outpatient-treatment-of-lower-respiratory-tract-infections-in-children-with-tracheostomy 